\title{
Recent results of the ANTARES neutrino telescope
}

\section{Tommaso Chiarusi on behalf of the ANTARES Collaboration*}

E-mail: tommaso.chiarusi@bo.infn.it

The ANTARES detector is the first Cherenkov neutrino telescope realised in the Mediterranean sea. It is continuously taking data since 2007 , with the primary aim to detect astrophysical neutrinos in the TeV-PeV range. A very good angular resolution in all flavour neutrino interaction channels, together with the depth of the abyssal site (2500 m below the sea level) led to an unprecedented sensitivity in the searches for neutrino sources in the Southern Sky and in the energy range below $100 \mathrm{TeV}$. This has allowed constraining on the origin of the cosmic neutrino flux discovered by the IceCube detector. ANTARES is also focussed on a rich multi-messenger program, providing with both online and offline actions or feedbacks. Among these, the searches triggered by gravitational wave observations are of prominent interest. Other physics topics are also covered: searches of dark matter annihilation or decay in massive objects; the search for relic massive magnetic monopoles and nuclearites; the study of atmospheric neutrinos and neutrino oscillations.

European Physical Society Conference on High Energy Physics - EPS-HEP2019 -

10-17 July, 2019

Ghent, Belgium

${ }^{*}$ Speaker. 


\section{Introduction}

Since 2013, after their first detection by IceCube [1], astrophysical neutrinos gained their place, together with cosmic-rays and photons, as messengers for the most violent phenomena in the Universe. Neutrinos can emerge from dense media and travel across cosmological distances without being deflected by magnetic fields nor absorbed by ambient matter and radiation. Multi$\mathrm{TeV}$ neutrinos are expected to be emitted in a large sample of astrophysical objects: together with gamma-rays, they are produced in the interactions of accelerated nuclei with matter and radiation at the sources or along their acceleration path. Neutrino Astronomy is nowadays part of the multimessenger approach, which includes gravitational waves and also other form of radiations. However the identification of neutrino sources is still missing. In order to achieve this goal, larger data samples are required, provided by improved detectors with a better angular resolution and interconnected to grant a full sky coverage. ANTARES and KM3NeT neutrino telescopes play a major role for complementing the IceCube searches at South Pole. As installations in the Mediterranean Sea, they benefit of optimal optical properties of seawater which allow unprecedented pointing and reconstruction performance. In this contribution, we report the status of the ANTARES detector, still operating since more than 12 years, with its main scientific achievements.

\section{The ANTARES telescope: detector and performances}

The ANTARES (Astronomy with a Neutrino Telescope and Abyss environmental Research) detector [2] is the largest neutrino telescope operating undersea. It is installed at a depth of $2475 \mathrm{~m}$ in the Mediterranean Sea, about $40 \mathrm{~km}$ off the French coast near Toulon (France), and is running since 2007. It consists of an array of 12 mooring lines mutually distant of about $70 \mathrm{~m}$ and connected to a junction box which distributes the electrical power and transmits the data to shore through an electro-optical cable. Each line supports 25 storeys made of triplets of 10-inch photomultipliers (PMTs) enclosed in glass spheres, oriented at $45^{\circ}$ downwards. The detector comprises a total of 885 optical modules (OMs), corresponding to an instrumented volume of about $0,05 \mathrm{~km}^{3}$. ANTARES records the Cherenkov light emitted by the charged particles produced in the interaction of a high energy (HE) neutrino in the vicinity of the detector. Events are classified in two topology classes: track-like events are associated essentially to $v_{\mu}$ charged-current interactions producing a muon that crosses the detector, while cascade-like events correspond to all other (charged- and neutralcurrent) interaction channels, where only hadronic and electromagnetic showers are produced. The time, position and amplitude of the recorded signals on the PMTs are used to reconstruct the energy and the arrival direction of the incident neutrino. The optical properties of sea water allow excellent timing information for the detected light signals, yieding a very good angular resolution for the reconstructed direction of detected neutrino candidates for all event topologies $\left(\sim 0.4^{\circ}\right.$ for tracks above $10 \mathrm{TeV}$, Figure 1a, and $\sim 3^{\circ}$ for showers, Figure 1b). Such reconstruction performance also relies on the detector accuracy in the measurement of the hits arrival time $(\sim 1 \mathrm{~ns})$ and position $(\sim$ $10 \mathrm{~cm}$ ). Time calibration is performed by means of an insitu array of laser and LED beacons. The position of the OMs is continuously monitored thanks to a high-frequency long-baseline acoustic network comprising fixed emittors deployed on the seabed, and hydrophones distributed along the detector lines, whose position is determined by triangulation based on travel time measurement 


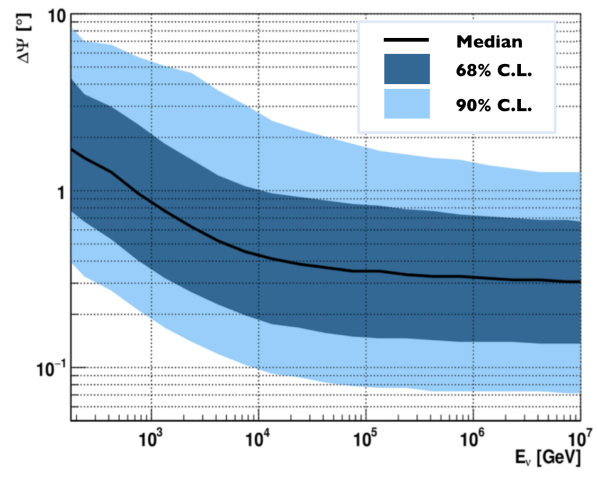

(a)

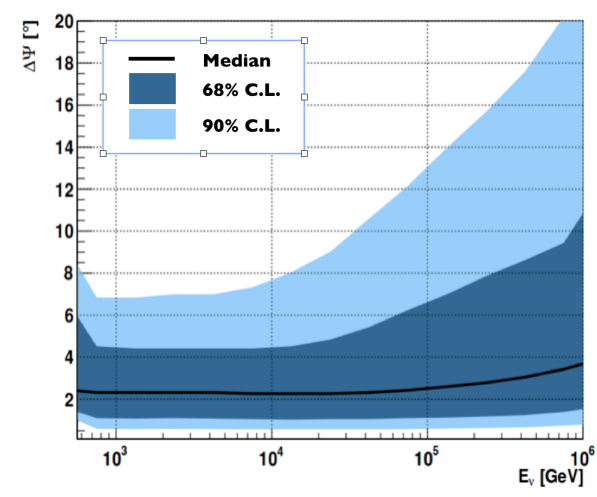

(b)

Figure 1: ANTARES angular resolution for track-like events (a) and for cascade-like ones (b).

of acoustic waves. A tiltmeter and a compass enclosed in the local electronics module of each storey allow for the reconstruction of its exact orientation. The absolute pointing performance of the detector has been validated by measuring the deficit of atmospheric muons in correspondance with the direction of the Moon, the so called Moon shadow, by analysing the data collected in the period 2007-2016 corresponding to a livetime of 3128 days. The Moon shadow was observed with a statistical significance of $3.5 \sigma$ [3]. Complementary oceanographic measurements are performed on a dedicated instrumented line, in particular to retrieve the sound velocity and to measure the sea current $(\mathrm{O}($ few $\mathrm{cm} / \mathrm{s}))$.

\section{Diffuse flux searches}

A neutrino diffuse flux can originate from the ensemble of unresolved individual sources, too faint to be detected, by cosmic ray propagation effects or by a combination of these processes. The IceCube findings mentioned in the opening of these proceedings concern to a highly-significant excess compatible with the hypothesis of an all-flavour neutrino flux isotropically distributed over the whole sky. Although the measured energy spectra fits to an unbroken single-power-law of the kind $d \Phi(E) / d E=\Phi_{0} E^{-\Gamma}$, some tension is present between the results obtained independently with the two principal data samples: the High Energy Starting Events (mainly cascades pointing to the Southern sky) producing a softer spectrun $(\Gamma \sim 2.5)$ and the Track-like Events ( upwardgoing muons from the Nother sky) giving a harder spectrum $(\Gamma \sim 2.1)$.

ANTARES events, collected during 11 years of data taking from 2007 and 2018 [4] offer the opportunity to perform a complementary search. After energy-related selection cuts, a total of 50 ( 27 track-like +13 shower-like) events are observed in data, with $36.1 \pm 8.7$ being expected from pure background. A maximum-likelihood method is applied to fit the observed distributions of the energy estimators. The best-fit cosmic flux yields a single-flavor normalization at $100 \mathrm{TeV}$ equal to $\Phi_{0}^{1 f}(100 \mathrm{TeV})=1.5 \pm 1.0 \times 10^{-18} \mathrm{GeV}^{-1} \mathrm{~cm}^{-2} \mathrm{~s}^{-1} \mathrm{sr}^{-1}$ and a spectral index $\Gamma=2.3_{-0.4}^{+0.4}$, as shown in Figure 2a. Although the null cosmic flux hypothesis can't be excluded with a large significance $(1.8 \sigma)$, the ANTARES result is inline with the hypothesis that the IceCube flux has a cosmic origin. 
The "KRA $\gamma$ " model [5] accounts for local cosmic ray flux measurements as well as Galactic $\gamma$-ray observations. It seems to favour the possibility for some detectable neutrino events coming from the interaction of cosmic rays with the interstellar medium. Such a model enforced the speculations on a potential neutrino Galactic component rised in [6]. For this reason a joint analysis was performed, combining the 7 years track sample of IceCube and the 10 years track and cascades events recorded by ANTARES [5]. No significant excess was found to justify the mentioned "spectral anomaly" between the Southern and Northern emisphere. Limits are sets as shown in Figure $2 b$.

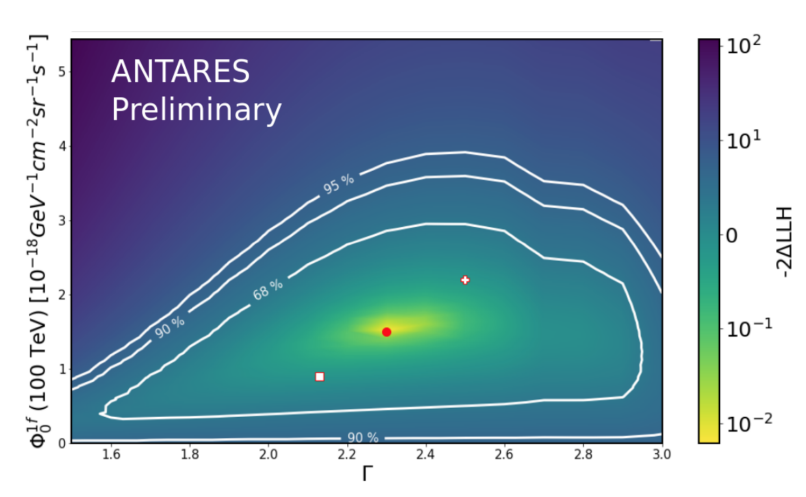

(a)

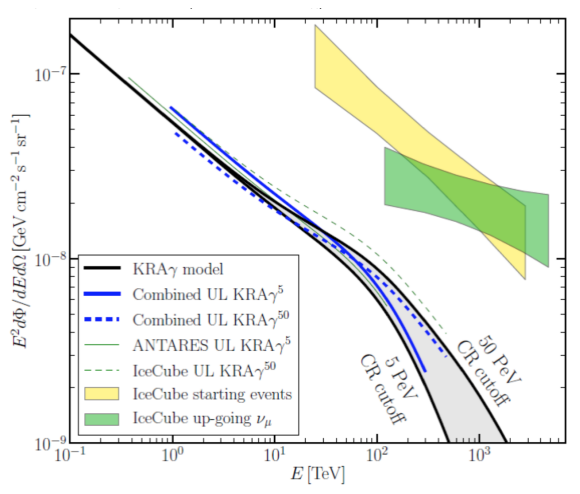

(b)

Figure 2: (a) 2D likelihood landscape of the parameter fitting for the spectral index $\Gamma$ and the one-flavour flux normalisation at $100 \mathrm{TeV} \Phi_{0}(100 \mathrm{TeV})$; the ANTARES best fit point is shown as a red circle. Also shown the IceCube best fits from the combined all-sky HESE sample (empty cross) and the only Northern-sky upwardgoing munons (empty square) analises, respectively from ref. [7] and [6]. The white countours show the $68 \%, 90 \%$ and $95 \%$ confidence areas. (b) IceCube and ANTARES $90 \%$ upper limits according to individual (green dashed and continue lines) and combined (blue lines) analyses, according to the 3-flavor neutrino flux of the KRA $\gamma$ model (5-50 PeV cutoff). The combined analysis excludes at $90 \%$ C.L. the KRA $\gamma^{50}$.

\section{Point sources searches}

The reported excellent angular resolution, both for muon tracks and for electromagnetic and/or hadronic showers, allowed to extend the point-source search to the all-flavour scenario. The latest ANTARES search for point-like neutrino sources makes use of events recorded in 11 years of datataking, between early 2007 and the end of 2017 [8]. The selected sample consists of 8754 tracks and 195 showers. Three different searches for cosmic neutrino sources are performed: a scan over the whole ANTARES visible sky, an investigation of 112 astrophysical candidates and 75 IceCube tracks. No significant evidence of cosmic neutrino sources is found. The sensitivity of this analysis versus the source declination is shown in Figure 3a, together with the 90\% C.L. upper limits on the neutrino flux from the investigated astrophysical candidates. The predictions for KM3NeT/ARCA [9] are also shown. KM3NeT/ARCA is expected to exceed the current ANTARES sensitivity to point-like sources by a factor of $\sim 20$ after six years of operation [10]. The 11-years ANTARES 
data set has been also used to look for a steady emission of neutrinos from the direction of the blazar TXS 0506+056 [11], being considered the first high-energy neutrino source ever found, as published by IceCube in [12]. No significant evidence of cosmic neutrinos is found, with a total number of fitted signal events $\mu_{\text {sig }}=1.03$ at the location of the blazar and a p-value of $3.4 \%$ for an unbroken power-law spectrum $E^{-2.0}$. One track-like event mostly influences the fit (see Figure $3 \mathrm{~b}$ ). It occurred on December $12^{\text {th }}, 2013$, and is located within $1 \sigma$ from the source position. The value of the energy estimator for this event is such that only $9 \%$ of the neutrino candidates inducing a track have a larger value. From these null results, 90\% C.L. upper limits on the flux normalization factor are derived.

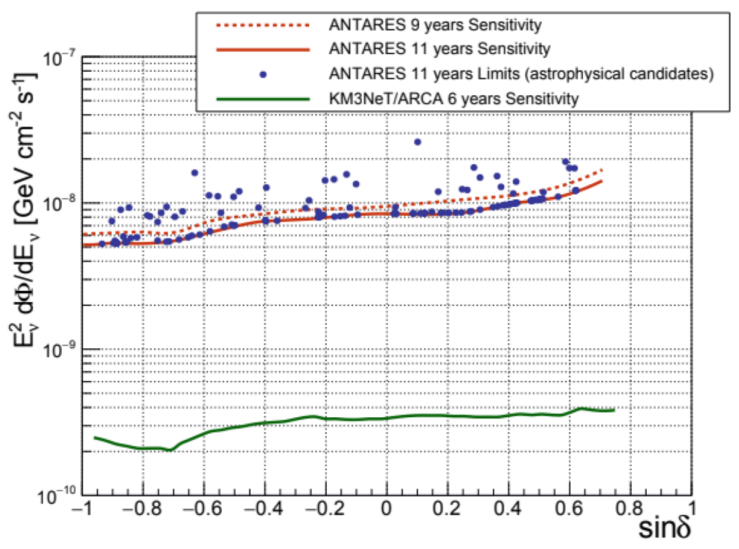

(a)

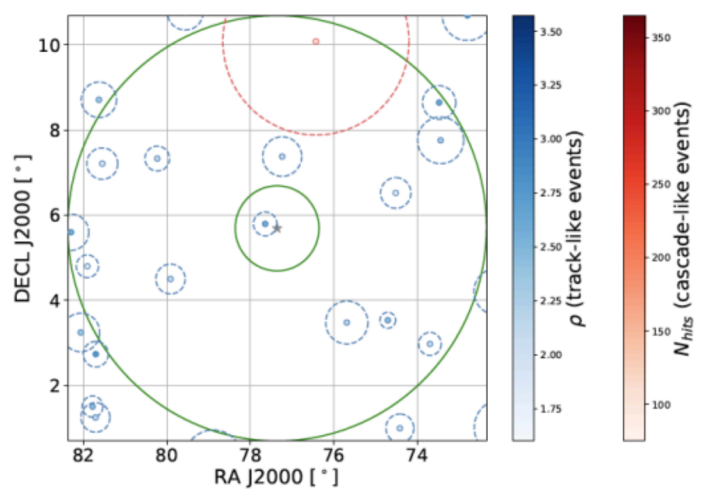

(b)

Figure 3: (a) ANTARES 9- and 11-years sensitivity to point-like sources and upper limits for the investigated astrophysical candidates versus the source declination for an $E^{-2}$ spectrum. For comparison, 6-years sensitivity for KM3NeT/ARCA is also reported. (b) Distribution of events in the equatorial coordinates at the location of TXS 0506+056. The red points denote shower-like events, whereas the blue points indicate track-like events. The dashed circles around the events indicate the angular error estimate. Different tones of red and blue correspond to the values assumed by the energy estimators.

\section{Dark matter searches}

The Weakly Interactive Massive Particles (WIMPs) are dark matter candidates. Their signature can be an excess of neutrinos from the direction of massive astrophysical objects like the Sun, the Milky Way centre, the centre of the Earth, some dwarf galaxies and galaxy clusters. Due to the environment gravitation pull and the scattering with ordinary matter, WIMPs are expected to accumulate, annihilate in pairs and subsequently produce standard model particles, including neutrinos. The Sun and the Galactic Centre represent two particularly interesting dark matter source candidates for ANTARES (and in the future also for KM3NeT) since the latitude of the detector location offers an observation of the Sun with less atmospheric background and a privileged visibility of the Galactic Centre. Figures $4 \mathrm{a}$ and $4 \mathrm{~b}$ show the results of the latest ANTARES searches for WIMP-annihilation induced neutrinos, respectively from the direction of the Sun [13] and of 
the Galactic Centre [14], compared to those of other experiments. In Figure 4a, the most competitive limits on the spin-dependent (WIMP-proton) interaction cross section $\sigma_{p}^{S D}$ for $M_{W I M P}>200$ $\mathrm{GeV}$ is provided by the active neutrino telescopes. Such limits will be extended to lower WIMP masses by the future low-energy threshold detector KM3NeT/ORCA [9]. As the performances of KM3NeT/ARCA are currently under evaluation, the high-energy detector is expected to provide further improvements especially for large values of $M_{W I M P}$. In Figure $4 \mathrm{~b}$ one can see that the limits on the WIMP-WIMP velocity- averaged self-annihilation cross section $\langle\sigma v\rangle$ set by ANTARES are the most stringent ones among those from neutrino telescopes.

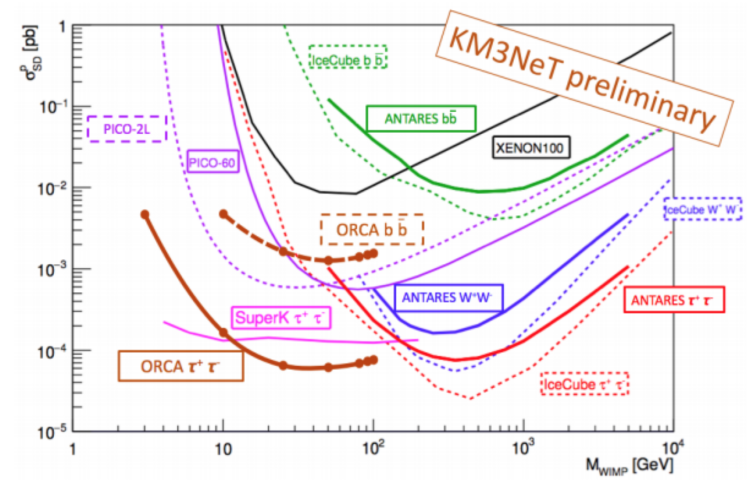

(a)

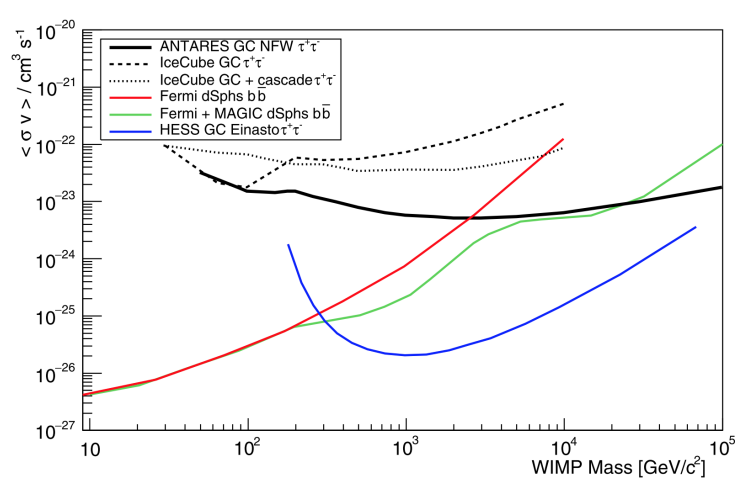

(b)

Figure 4: $90 \%$ C.L. limits on the thermally averaged annihilation cross-section $\langle\sigma v\rangle$ in the Sun (a), and on the spin- dependent (WIMP-proton) interaction cross section $\sigma_{S D}^{p}$ for different annihilation channels from the Galactic Centre (b), as a function of the WIMP mass.

\section{The multi-messenger program}

The ANTARES Collaboration has also developed an active multi-messenger program devoted to correlate neutrino events - in space and/or time - with electromagnetic, cosmic ray and gravitational waves counterparts. Specific low-latency pipelines have been implemented for distributing alerts to optical robotic telescopes (TAROT, ROTSE and MASTER), radio (MWA), X-ray (Swift, INTEGRAL) and $\gamma$-ray (H.E.S.S., HAWC) telescopes. Dedicated analyses in ANTARES also follow-up on external alerts distributed through the Gamma-ray Coordinated Network to look for transient events. Several on- and off-line studies have also been conducted to search for neutrinos in coincidence with the gravitational wave events recently detected by LIGO and Virgo [15, 16, 17]. It is worthy to mention three follow-up analyses that were performed between 2017 and 2018, initially triggered by the GCN circular [18], reporting about the extremely high energy cosmic neutrino IC170922A. None of the three analyses (online, time independent and time dependent point-source approaches) yielded neutrino events, as reported in [11]. Recently, an ANTARES study aimed at constraining a possible origin of the IceCube astrophysical signal from transient sources [19]. The ANTARES data-set was scanned to look for time and space correlation with 54 IceCube track-like events. Each IceCube event was treated as a potential transient neutrino source. Althought neutrino arrival times were used to enhance the discovery potential, no ANTARES event 
was observed in correlation with the IceCube candidates, and upper limits on the neutrino fluence from the investigated candidates were set. Finally, ANTARES has also looked for neutrinos in coincidence with 12 Fast Radio Bursts [20] : also in this case no coincident neutrino candidate is observed, and constraints on the neutrino fluence and on the energy released are derived from the associated null results.

\section{Conclusions}

The ANTARES detector has been continuously taking data since more than 12 years. The obtained science results are competitive and contribute significantly to a wide range of Astrophysics and Physics searches. In addition to the topic reported in these Proceedings, it is worthy also to mention the recent atmospheric neutrino oscillation searches [21] and the magnetic monopole searches [22]. The complementarity of ANTARES with respect to IceCube demonstrates the huge potential of KM3NeT, the next generation Mediterranean Cherenkov telescope, expected to open a new era of neutrino Astronomy.

\section{References}

[1] M. G. Artsen et al., Science 342 (2013) .

[2] M. Ageron et al., Nucl. Instrum. Meth. A 656 (2013) 11.

[3] A. Albert et al., Eur.Phys.J. 78 (2018) 1006.

[4] L. A. Fusco, F. Versari (ANTARES Collaboration) PoS(ICRC2019) (2019) 981.

[5] D. Gaggero et al., The Astrophysical Journal Letters 815 (2015) L25.

[6] M. G. Artsen et al., Astrophysical Journal 833 (2016) 3.

[7] M. G. Artsen et al., Astrophysical Journal 809 (2015) 98.

[8] J. Aublin, G. Illuinati, S. Navas (ANTARES Collaboration) PoS(ICRC2019) (2019) 920.

[9] C. Distefano, (KM3NeT Collaboration) in these Proceedings.

[10] R. Coniglione,(ANTARES and KM3NeT Collaboration) PoS(ICRC2019) (2019) 920.

[11] A. Albert et al.The Astrophysical Journal Letters 863 (2018) L30.

[12] M. G. Artsen et al., Science 361 (2018) 147.

[13] S. Adrián-Martínez et al.Physics Letters B 759 (2016) 69.

[14] A. Albert et al., Phys. Lett. B 796 (2019) 253.

[15] A. Albert et al.The Astrophysical Journal Letters 850 (2017) L35.

[16] A. Albert et al.Phys. Rev. D 96 (2017) 022005.

[17] S. Adrián-Martínez et al.Phys. Rev. D 93 (2016) 122010.

[18] GCN circular 21916, 2017.

[19] A. Albert et al.The Astrophysical Journal 879 (2019) 108.

[20] A. Albert et al., Mon. Not. Roy. Astron. Soc. 842 (2019) 184.

[21] A. Albert et al.J. High Energ. Phys. 2019 (2019) 113.

[22] A. Albert et al.J. High Energ. Phys. 2017 (2017) 54. 